\title{
LOS NUEVOS USOS TURÍSTICOS EN LA RESERVA DE LA BIOSFERA DE LA ISLA DE LA PALMA (CANARIAS)
}

\author{
Alejandro González \\ José Ángel Hernández \\ Juan Manuel Parreño \\ Departamento de Geografía. Universidad de Las Palmas de Gran Canaria \\ agonzalez@dgeo.ulpgc.es, jose.hernandez@ulpgc.es, jparreno@dgeo.ulpgc.es
}

\section{RESUMEN}

En el presente trabajo abordamos el estudio de la Reserva de la Biosfera de la isla de La Palma y de la dinámica de los nuevos usos turísticos que se están implantando con la finalidad de reflexionar sobre el modelo de desarrollo que implican. En el estudio se realiza una breve diagnosis de las principales características del modelo territorial de la isla. Con posterioridad, se analiza el caso de la Reserva de la Biosfera de la Isla Bonita, sus características y sus objetivos. Por último, se analiza el modelo turístico que se está implantando desde los instrumentos de ordenación territorial con el fin de valorar su nivel de sostenibilidad y de adecuación a los principios de la Reserva.

Las fuentes consultadas han sido los informes de la Reserva de la Biosfera, los documentos de planificación territorial de la isla, asociaciones de desarrollo rural, las concejalías de desarrollo rural de algunos ayuntamientos implicados y las consejerías de turismo y agricultura del Cabildo Insular de La Palma y del propio Gobierno de Canarias.

Palabras clave: Reserva de la Biosfera, desarrollo sostenible, modelo territorial, usos turísticos, estructura demográfica.

Fecha de recepción: febrero 2014.

Fecha de aceptación: diciembre 2014. 


\section{ABSTRACT}

In this paper we study the Biosphere Reserve on the island of La Palma and the dynamics of new tourist uses that are being implemented in order to reflect on the development model involved. The study consists of a brief diagnosis of the main features of the territorial model of the island. Subsequently, we analyze the case of the Biosphere Reserve of La Isla Bonita, their characteristics and objectives. Finally, we analyze the tourist model which is being implemented from the planning instruments in order to assess their level of sustainability and adaptation to the principles of the Reserve.

The sources consulted were the reports of the Biosphere Reserve, territorial planning documents of the island, rural development associations, the rural development departments of some municipalities and the regional ministries of tourism and agriculture of the Cabildo of La Palma and Government of The Canary Islands.

Keywords: Biosphere Reserve, sustainable development, territorial model, tourist uses, demographic structure.

\section{INTRODUCCIÓN}

Las Reservas de la Biosfera surgen en 1968 en el marco del programa MAB (Hombre y Biosfera) de la UNESCO con la intención de favorecer la conservación de la diversidad de la vida en la Tierra. Por tanto, en un primer momento se declaran como Reservas de la Biosfera espacios de alto valor natural sin apenas ocupación humana. En la medida en que los modelos de desarrollo sostenible se han ido desarrollando a lo largo de los ochenta y noventa, el concepto ha ido evolucionando declarándose enclaves de gran valor natural que además presentan un nivel moderado de transformación antrópica. Hoy en día, las Reservas de la Biosfera son zonas terrestres, costeras o marinas en las que se pretende poner en marcha un modelo de desarrollo sostenible que compatibilice el desarrollo económico y social con la conservación de sus valores naturales.

La Palma, una de las más occidentales de las Islas Canarias, situada en torno al paralelo $28^{\circ} 40^{\prime}$ y el meridiano $17^{\circ} 52^{\prime}$, y con una extensión de $708 \mathrm{~km}^{2}$ y 2.426 metros de altitud, presenta valores naturales geológicos, geomorfológicos y biogeográficos. Así por ejemplo, destaca en esta isla la última erupción subaérea que ha tenido el Archipiélago Canario, esto es el volcán Teneguía de 1971; tiene una magnífica representación del bosque de laurisilva en la vertiente de barlovento) como para que una parte de su territorio fuera declarada en 1983 como Reserva de la Biosfera. En concreto, 511 has en El Canal y Los Tiles, en el municipio de San Andrés y Sauces, fueron consideradas de este modo por la diversidad y la conservación de sus bosques de laurisilva. Desde entonces, la Reserva ha ido creciendo en la medida en que iba evolucionando el concepto, con el fin de posibilitar la implantación de un modelo de desarrollo sostenible. De este modo, en 1988, se amplía la superficie protegida a 13.240 has y en 2002, se acuerda denominar la totalidad del territorio insular como Reserva Mundial de la Biosfera.

El modelo productivo de la isla se había basado hasta hace unas décadas en la producción agraria, esencialmente en los cultivos de exportación (plátanos) y en el desarrollo de una 
agricultura de subsistencia. La falta de viabilidad de este modelo ha orientado el sistema productivo hacia el sector servicios y al desarrollo de la actividad turística. La declaración de la isla como Reserva de la Biosfera implica potenciar esta última opción en la medida en que este tipo de calificaciones actúan como reclamo turístico, al mismo tiempo que estimula un modelo turístico compatible con los valores ambientales, culturales y paisajísticos de la isla.

Hasta ahora la actividad turística se ha centrado en fórmulas como el turismo rural o el ecoturismo y en el desarrollo de algunos enclaves costeros como turismo de masas, sin que el volumen de turistas recibidos haga peligrar los valores naturales de la Isla. La aprobación de la Ley 19/2003, de 14 abril, de las Directrices de Ordenación General y las Directrices de Ordenación del Turismo de Canarias y de la más reciente Ley 6/2009, de 6 de mayo, de medidas urgentes en materia de ordenación territorial para la dinamización sectorial y la ordenación del turismo han puesto las bases para una intensificación del uso turístico en el suelo insular (Parreño y Díaz, 2010). En este contexto, cabe preguntarse, cuando aún estamos al principio de lo que parece ser un nuevo modelo turístico en La Palma, hasta qué punto éste será compatible con los principios y objetivos de la Reserva de la Biosfera.

El objetivo de este trabajo es, por tanto, analizar si la actividad turística que se quiere implantar en la isla de La Palma contribuirá a consolidar un modelo de desarrollo sostenible coherente con los objetivos que plantea la declaración de la isla como Reserva de la Biosfera, o si por el contrario, supone introducir la isla en el mercado turístico de masas.

Para esta finalidad, las fuentes consultadas han sido los informes de la Reserva de la Biosfera, los documentos de planificación territorial de la isla, las asociaciones de desarrollo rural de la Isla, en particular ADER - La Palma, las concejalías de desarrollo rural de los ayuntamientos implicados, así como las consejerías de turismo y agricultura del Cabildo Insular y del propio Gobierno de Canarias.

En el trabajo se realiza una diagnosis o análisis de los factores que han contribuido a formar estos espacios, tanto los de carácter natural (paisaje biofísico: geomorfología y biogeografía), como los factores socioeconómicos (población, economía, etc.), aunque por razones de espacio, no desarrollamos éstos con gran exhaustividad. Posteriormente, se analiza cómo se ha estructurado y que supone la declaración de Reserva de la Biosfera. Por último se analiza la propuesta de los nuevos usos turísticos que introduce la planificación territorial de la isla con el fin de valorar su sostenibilidad y la coherencia con los objetivos de la Reserva.

\section{BREVE MARCO NATURAL Y SOCIOECONÓMICO DE LA PALMA}

La isla de La Palma presenta un territorio de gran valor natural y paisajístico. Sin duda alguna, la flora y la fauna de la Isla es uno de los valores que más peso han tenido para que haya sida declarada Reserva de la Biosfera. Destaca la presencia de una extensa masa de bosque húmedo subtropical, mayoritariamente laurisilva en la vertiente de barlovento, entre 600 y 1200 metros de altitud, en muy buen estado de conservación. También podemos encontrar representaciones muy extensas del pinar canario por encima de esta altitud hasta los 1800 metros. A continuación, tenemos el dominio de la vegetación de cumbre (retamar - codesar y tagasaste, asociados a gramíneas y a especies herbáceas de bajo porte). A estos espacios vegetales le acompaña una fauna original con numerosos endemismos, entre los que destacamos las grajas, la paloma rabiche, la paloma torcaz, etc. 
En cambio la representación de la flora del piso basal (por debajo de los 200 metros) es escasa por coincidir con la zona de mayor antropización agrícola y de establecimiento de los principales núcleos de poblamiento. Esto mismo se puede afirmar para el piso de transición o de bosque termófilo, entre los 200 y 600 metros, con gran presencia de la agricultura y también, en menor medida, del poblamiento.

La isla también cuenta con un importante patrimonio volcánico, con manifestaciones recientes. De hecho presenta la última erupción histórica subaérea habida en el Archipiélago hasta la actualidad, es decir, la del volcán de Teneguía, cuya erupción tuvo lugar en 1971. También son notables las erupciones del Nambroque o San Juan (1949), con los cráteres del Duraznero, del Hoyo Negro y el de Llano del Banco; la del Charco (1712); la del volcán de San Antonio, en Fuencaliente (1677); la de Martín (1646); la de Tajuya, en El Paso (1585); y, por último, la de Tacande (1470). Igualmente, presenta un gran interés geológico y turístico la formación de la Caldera de Taburiente, una depresión erosiva de gran magnitud. También son muy llamativos sus barrancos, sobre todo los de la mitad septentrional, que es la parte más antigua y por consiguiente más erosionada. No tiene importantes playas de arena, pues su costa es mayoritariamente acantilada, salvo en las desembocaduras de los barrancos con algunas playas de callaos y arenas negras.

Por todo ello, La Palma cuenta con un paisaje espectacular. Su eslogan turístico más afamado es el de Isla Bonita, por la belleza que encierra buena parte del suelo insular, tanto la zona de montaña, como el borde de la Caldera de Taburiente, y en particular la zona del entorno del Roque de Los Muchachos.

No es por tanto extraño que la isla de La Palma cuente con una superficie de espacios naturales protegidos considerable $\left(247,9 \mathrm{~km}^{2}\right.$ de su extensión territorial, esto es, el $35 \%$ del total de la superficie insular) (Gobierno de Canarias, 2003). Aparte del Parque Nacional de Taburiente, creado en 1954, cuenta también con dos parques naturales: P. N. de Las Nieves y el P. N. de Cumbre Vieja; con ocho monumentos naturales: M. N. de Montaña Azufre, M. N. de Los volcanes de Aridane, M. N. del Risco de La Concepción, M. N. de la Costa de Hiscaguán, M. N. del Barranco del Jorado, M. N. de Volcanes de Teneguía, M. N. del Tubo Volcánico de Todoque; una reserva natural integral, en concreto el Pinar de Garafía; una reserva natural especial, la de Guelguén; cuatro paisajes protegidos: el del Tablado, Barranco de las Angustias, el de Tamanca y El Remo; y por último tres sitios de interés científicos: el de Juan Mayor, el del Barranco del Agua y el de Las Salinas de Fuencaliente (tabla 1).

Tabla 1

SUPERFICIE Y NÚMERO DE ESPACIOS PROTEGIDOS DE LA PALMA

\begin{tabular}{|l|c|c|}
\hline Tipo espacio protegido & $\mathrm{N}^{\circ}$ & Superficie (Has.) \\
\hline Parque Nacional & 1 & 4.690 \\
\hline Parque Natural & 2 & 12.594 \\
\hline Monumento Natural & 8 & 1.452 \\
\hline Reserva Natural Integral & 1 & 984 \\
\hline Reserva Natural Especial & 1 & 1.074 \\
\hline Paisajes Protegidos & 4 & 4.108 \\
\hline Total & 17 & 24.902 \\
\hline
\end{tabular}

Fuente: ISTAC. Gobierno de Canarias. Elaboración propia. 
Esta gran biodiversidad natural se relaciona con unos usos históricos del territorio, asociados a la actividad de la agricultura, tanto de exportación como de autoconsumo y de mercado interno; a la ganadería: óvidos, cápridos, bovinos, porcinos y equinos fundamentalmente; y a la explotación forestal, debido a la gran masa boscosa que presentaba. La industria y el resto del sector secundario, apenas cuentan con un papel relevante en la estructura económica insular, pues incluso la escasa que existía - en concreto la del tabaco- , ha disminuido su importancia e incluso ha desaparecido del tejido industrial de algunas comarcas. El terciario es el sector de mayor actividad, aunque el turismo es escaso y está orientado hacia una tipología rural y de naturaleza en su mayoría, aunque también hay núcleos de sol y playa (Los Cancajos, Puerto Naos y parte de la costa de Fuencaliente).

No obstante la débil economía de La Palma, la Isla ha sufrido un rápido proceso de antropización, debido sobre todo a la fuerte transformación del territorio volcado hacia la economía de exportación, fundamentalmente de la caña de azúcar, la vid y el plátano.

Las zonas de mayor antropización, y por consiguiente con mayores impactos paisajísticos, son los municipios de Santa Cruz de La Palma y de Los Llanos de Aridane, esto es el

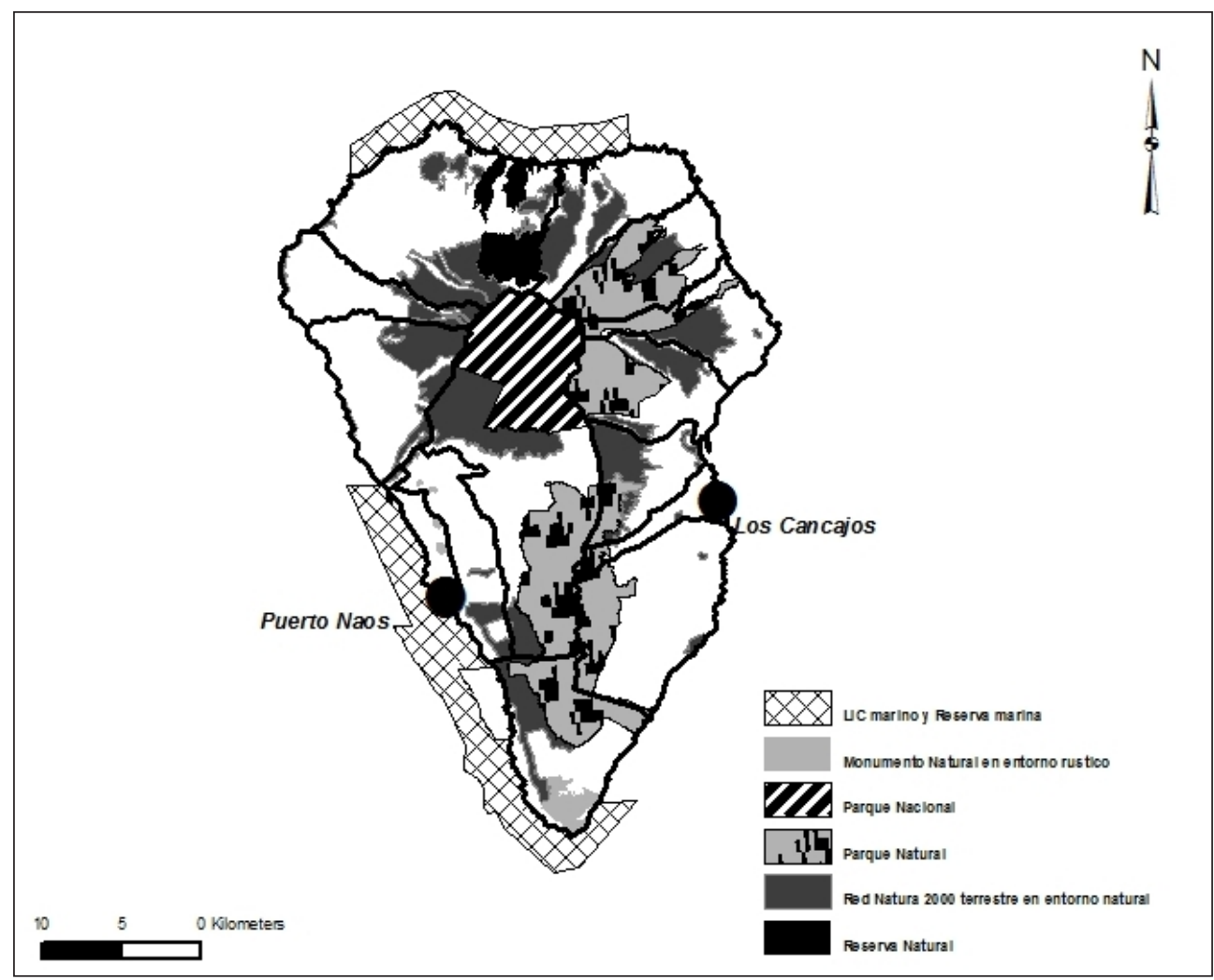

Fuente: elaboración propia. 
centro insular. En el municipio de Los Llanos de Aridane se localiza la mayor extensión de agricultura de exportación (plátanos sobre todo, y en menor medida aguacates y papayos). En cambio en Santa Cruz de La Palma se encuentra la capital insular, vinculada a una importante oferta de servicios. Hay que destacar que esta ciudad tiene uno de los cascos históricos mejor conservados y más interesantes de Canarias, pues no hay que olvidar que la capital palmera llegó a tener el tercer puerto más importante del imperio español en tiempos del rey Felipe II, tras Sevilla y Amberes.

En definitiva el modelo territorial actual de La Palma es el fruto de una particular riqueza de recursos naturales y de un no menos importante patrimonio cultural (Del Canto, 2000). Distinguimos cuatro grandes espacios: las zonas urbanas de los municipios antes señalados, los espacios turísticos de masas: Puerto Naos, en Los Llanos de Aridane; la costa suroeste del municipio de Fuencaliente, sobre todo la zona de Cerca Vieja; y el enclave de Los Cancajos, en Breña Baja (Figura 1); los espacios rurales de agricultura de exportación, costa de la mitad sur y suroccidental de la Isla, que abarca los municipios de Tazacorte, Los Llanos de Aridane y Fuencaliente, y en menor medida la costa este insular, desde Mazo hasta Barlovento; por último estaría el resto de La Palma con los espacios naturales protegidos y los rurales de agricultura y ganadería de autoconsumo y producción interior.

El modelo territorial no está exento de problemas ambientales y económicos. La sobreexplotación del suelo, del bosque y de los recursos en general ha hecho peligrar la sostenibilidad del propio sistema. No obstante y gracias al escaso crecimiento poblacional y del sector servicios, en particular el turismo, esta Isla no presenta graves impactos paisajísticos como sí se constatan en otras islas del Archipiélago. Sin lugar a dudas, lo más destacable son los graves problemas económicos del tejido productivo de la isla. El sector primario presenta una escasa rentabilidad y está en una situación de grave dependencia, en particular la agricultura del plátano y la cabaña ganadera. A ello se suma el declive de su agricultura de autoabastecimiento y del mercado interno y la falta de alternativas industriales. Todo ello repercute en un débil crecimiento demográfico en el conjunto insular y en proceso de pérdida de efectivos en determinados municipios, como se puede observar en la Tabla 2.

La evolución demográfica asociada a los cambios en la estructura económica, nos proporciona las claves para entender el modelo territorial actual y también las posibilidades futuras para alcanzar un modelo de desarrollo sostenible (Gómez Orea, D, 2002 y Gobierno de Canarias, 2008). Analizando qué zonas se han despoblado y por qué ocurre este fenómeno y estableciendo cuáles son los lugares con mayor dinamismo económico, podemos desentrañar las claves del modelo de explotación económica y de configuración territorial.

Los municipios de mayor población han sido tradicionalmente Santa Cruz de La Palma, capital insular, y los Llanos de Aridane (García Rodríguez, 1983) siendo éste último el principal motor económico por su potente agricultura de exportación, en particular el cultivo del plátano desde comienzos del siglo XX hasta la actualidad. Los municipios de economía rural tradicional han seguido perdiendo efectivos demográficos como consecuencia de las escasas expectativas económicas (Regidor, 2000) y por las mejoras de las infraestructuras viarias (Hernández Luis, 1993).

En general, se puede afirmar que La Palma ha terminado su transición demográfica, de un modelo demográfico tradicional, con abundante población joven y fuerte emigración, a otro con evidentes signos de envejecimiento. Por tanto, al exiguo contingente poblacional, se le 
suma una estructura por edad caracterizada por un reducido grupo de población joven como consecuencia de la progresiva disminución de la natalidad y de la emigración, un conjunto de población adulta no demasiado numeroso, ya que sus integrantes son los que se vieron más afectados por la movilidad hacia otros territorios, y un porcentaje de población anciana muy numerosa en relación al conjunto del Archipiélago.

Tabla 2

EVOLUCIÓN DE LA POBLACIÓN DE LA PALMA. 1900 - 2010

\begin{tabular}{|l|c|c|c|c|c|}
\hline Municipios & $\mathbf{2 0 1 0}$ & $\mathbf{2 0 0 1}$ & $\mathbf{1 9 6 0}$ & $\mathbf{1 9 4 0}$ & $\mathbf{1 9 0 0}$ \\
\hline Barlovento & 2.296 & 2.401 & 3.088 & 3.296 & 2.193 \\
\hline Breña Alta & 7.347 & 6.091 & 5.153 & 4.178 & 2.751 \\
\hline Breña Baja & 5.259 & 4.119 & 2.571 & 2.640 & 2.032 \\
\hline Fuencaliente & 1.898 & 1.794 & 2.140 & 2.314 & 1.783 \\
\hline Garafía & 1.714 & 2.012 & 4.915 & 5.068 & 3.244 \\
\hline Llanos de Aridane (Los) & 20.948 & 19.536 & 10.260 & 7.094 & 7.404 \\
\hline Paso (El) & 7.837 & 7.358 & 6.069 & 5.562 & 4.599 \\
\hline Puntagorda & 2.177 & 1.802 & 1.786 & 1.669 & 1.635 \\
\hline Puntallana & 2.425 & 2.258 & 2.570 & 2.634 & 2.252 \\
\hline San Andrés y Sauces & 4.874 & 5.263 & 7.113 & 5.798 & 3.659 \\
\hline Santa Cruz de La Palma & 17.128 & 18.206 & 13.380 & 11.013 & 7.383 \\
\hline Tazacorte & 5.697 & 6.117 & 4.689 & 3.942 & $-*$ \\
\hline Tijarafe & 2.769 & 2.741 & 3.029 & 3.281 & 2.963 \\
\hline Villa de Mazo & 4.955 & 4.621 & 5.247 & 5.555 & 4.605 \\
\hline LA PALMA & 87.324 & 84.319 & 72.010 & 64.044 & 46.503 \\
\hline
\end{tabular}

* La población de 1900 de Tazacorte estaba agregada al municipio de Los Llanos de Aridane, ya que el primero obtuvo su ayuntamiento en 1927.

Fuente: ISTAC. Elaboración propia.

En efecto, el grupo de población de más de 65 años supone un 19,3\% de la población total frente al 13,4\% del conjunto de Canarias. Por el contrario, la proporción de población joven, (14 y menos años), presenta un $13 \%$, frente al 14,7 \% del Archipiélago (Padrón de habitantes, 2010). De modo evidente, ello demuestra que la economía de La Palma es escasamente atrayente para los jóvenes $y$, por ende, que la isla presenta un alto grado de envejecimiento.

Desglosando los datos por municipios, prácticamente todos presentan un notable envejecimiento, aunque hay diferencias significativas entre los que tienen actividad turística y agricultura de exportación (por ejemplo Los Llanos de Aridane), y los que siguen sumidos en unas estructuras agrarias tradicionales (es el caso de Garafía).

En síntesis, La Palma presenta un modelo productivo que no es capaz de impulsar el crecimiento y el rejuvenecimiento de la población, al mismo tiempo que mantiene gran parte 
de sus valiosos recursos naturales, culturales y paisajísticos en buen estado de conservación. Esta situación es la que justifica que en las últimas décadas se haya apostado por desarrollar un nuevo modelo productivo para la isla. En este contexto hay que situar la declaración de la isla como Reserva de la Biosfera.

\section{LA RESERVA DE LA BIOSFERA DE LA PALMA}

La Isla Bonita cuenta con esta denominación desde 1983 aunque incluye a la totalidad de la isla desde $2002^{1}$. Desde entonces, la reserva está conformada por tres zonas diferenciadas (Fig. 2):

a. Zona núcleo, que engloba los sistemas menos explotados, abarcando el interior de la Caldera de Taburiente, la banda de las medianías del norte insular, la estribación montañosa de Cumbre Vieja, y la zona ocupada por las lavas del volcán histórico del San Juan (Nambroque, 1949), conocida como Las Manchas.

b. Zona tampón, que envuelve y protege a la zona núcleo.

c. Zonas exteriores de transición, en la cual se favorece el uso de los recursos y que constituye el resto de la Isla.

La declaración de la totalidad de la isla como Reserva de la Biosfera le ha proporcionado a la Isla un distintivo de calidad ambiental innegable, al mismo tiempo que supone una apuesta por un modelo de desarrollo sostenible que permita corregir los desequilibrios y debilidades económicas, los problemas demográficos y los desajustes territoriales. De hecho, según el Marco Estatutario de las Reservas de la Biosfera (Bilbao, 2007) éstas deben contar, además de con una notable biodiversidad y medidas para su protección, con un programa de intervención que aproveche sus fortalezas y oportunidades en pos de un modelo económico sostenible.

En el caso de La Palma, la apuesta por la sostenibilidad se centra en el desarrollo de los tipos de turismo que estén en consonancia con los recursos que dispone la isla (turismo rural, agroturismo, natural o ecoturismo, cruceros y cultural) y de la actividades agrarias.

En este sentido, hay que entender las principales propuestas realizadas por ADER (ADER, 2007) para aprovechar los recursos existentes:

$1^{\circ}$. Intervenir en los servicios y equipamientos de los distintos núcleos de población que están insertos en las áreas más deprimidas de la Reserva de la Biosfera y así evitar su despoblamiento (Mendez, R, 1994).

$2^{\circ}$. Creación y promoción de empleo rural asociado a las nuevas modalidades turísticas ya que pueden contribuir a frenar el despoblamiento y a fijar a los jóvenes a su lugar de origen o nacimiento, incidiendo en la reducción del envejecimiento demográfico. Fórmulas como el senderismo, el agroturismo y las visitas guiadas a los espacios naturales y protegidos pueden contribuir de forma poderosa a ello, incrementando el

1 La Palma fue la primera de las Islas Canarias en contar con una Reserva de la Biosfera. La segunda isla en conseguir este galardón de calidad fue Lanzarote en 1993. Unos años más tarde (2000) se concede el título a El Hierro. En cuarto lugar, se declara a una sexta parte de la isla de Gran Canaria (2005). En 2009, se incorpora la isla de Fuerteventura. Ésta y Gran Canaria no sólo tienen superficie protegida en el ámbito terrestre sino también el medio marino (VV. AA, 2006). Por último, en 2011, se le concede también el título a La Gomera. 
número de visitantes a la Isla y lo que es más importante, la capacidad de gasto de los mismos. Para ello es necesario una mayor profesionalización y cualificación de la población local, por lo que es necesario que se impartan cursos de formación y de promoción del empleo rural (Duarte, 2000; Bustos, 2006).

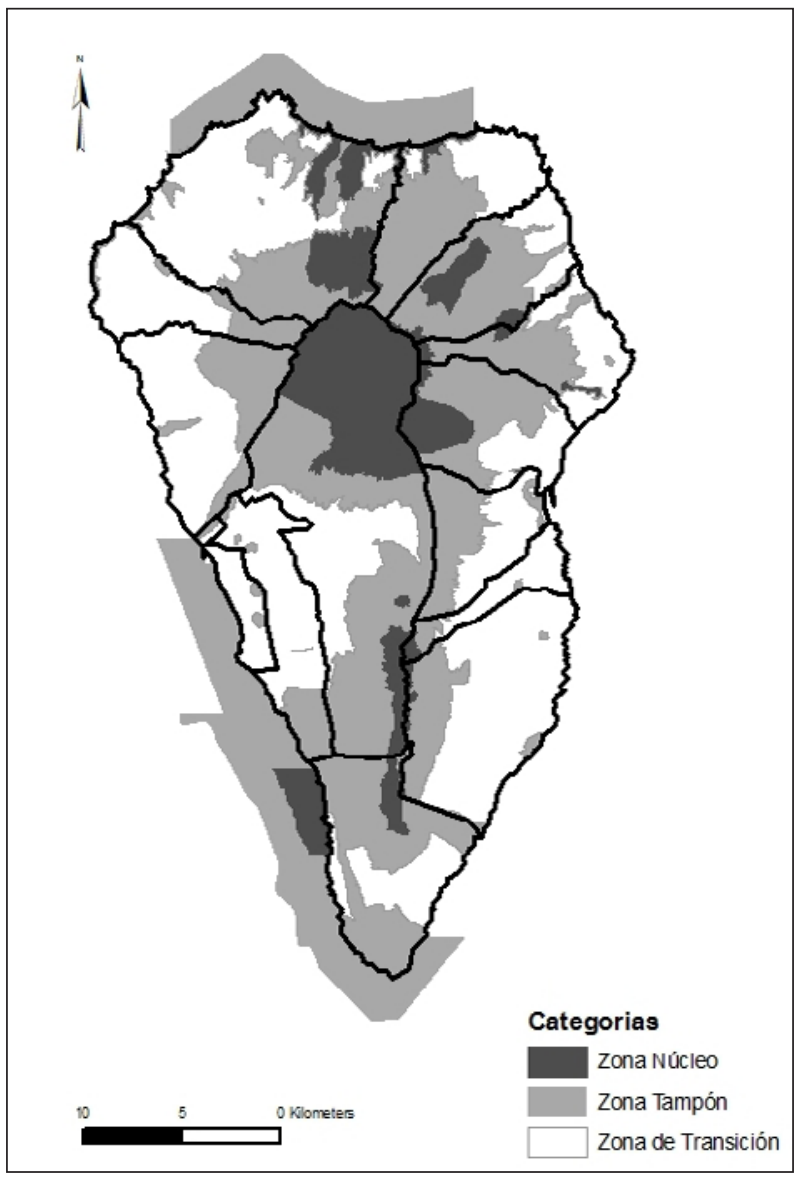

Fuente: ADER-La Palma.

$3^{\circ}$. Recuperación de la agricultura de medianías y de cumbres, tanto para mantener los puestos de trabajo y la población en las zonas rurales, como para evitar el éxodo rural. Esto permitiría a su vez el mantenimiento del paisaje agrario tradicional, constituyendo éste un valor tan importante como el propio paisaje natural, pues ambos consiguen una mayor calidad ambiental para este espacio insular. Todo ello está relacionado con el incremento de las producciones propias y así reducir la tasa de dependencia del exterior. Estos productos también constituyen magníficas oportunidades 
para vender a los turistas, suponiendo ello un ingreso complementario notable. No sólo se debe trabajar en la fase de producción, sino también en la más adecuada comercialización de los productos (vino, queso, miel, repostería de la almendra,...) (Gobierno de Canarias, 2005).

$4^{\circ}$. Mejora de la ganadería, pues sus productos tienen buenas posibilidades de colocarse en los mercados locales y entre las mercancías a consumir por los turistas (queso, carne, artesanía del cuero, etc.). Hay que mejorar las condiciones de explotación de la cabaña ganadera insular, con mejoras higiénicas y sanitarias en los corrales y establos, potenciar el consumo de forrajes locales, e introducir nuevos productos como pasteurizar la leche de cabra, realizar yogures, etc. Todo ello redundaría en beneficio del subsector ganadero y en la estabilidad del empleo en el campo, por tanto también contribuiría a mantener los valores medioambientales de los paisajes rurales.

$5^{\circ}$. Puesta en valor de los recursos forestales en las zonas de medianías y cumbres de la Isla para el desarrollo de la actividad turística. La explotación recreativa y de ocio de estos recursos es de gran interés para los turistas de senderos y rurales (Gobierno Vasco, 2008), pero también para las otras modalidades turísticas que presenta La Palma (ver figuras $3,4,5$ y 6 ).

Con todo ello se estaría contribuyendo a frenar la desagrarización de los espacios rurales y evitar así una excesiva terciarización de la economía insular, pero también se estarían incrementado las oportunidades para una explotación más sostenible del turismo en la Isla (Mosca y Ramos, 1994).

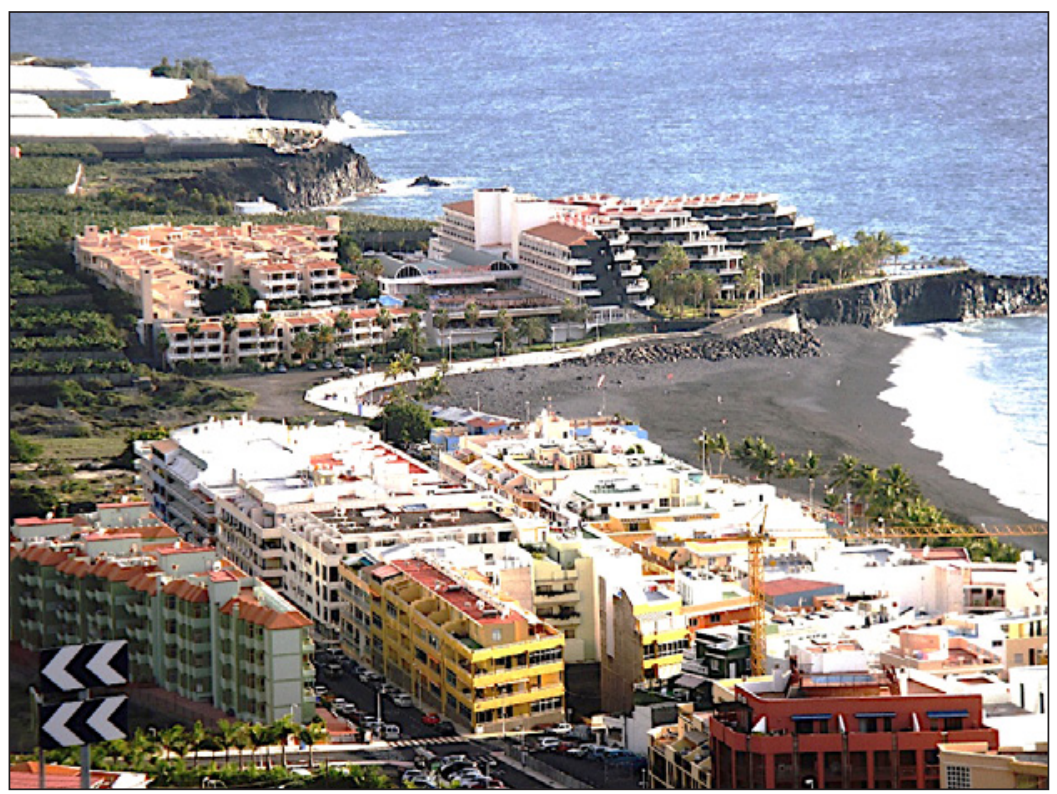


Figura 4

NÚCLEO TURÍSTICO DE LOS CANCAJOS (BREÑA BAJA)

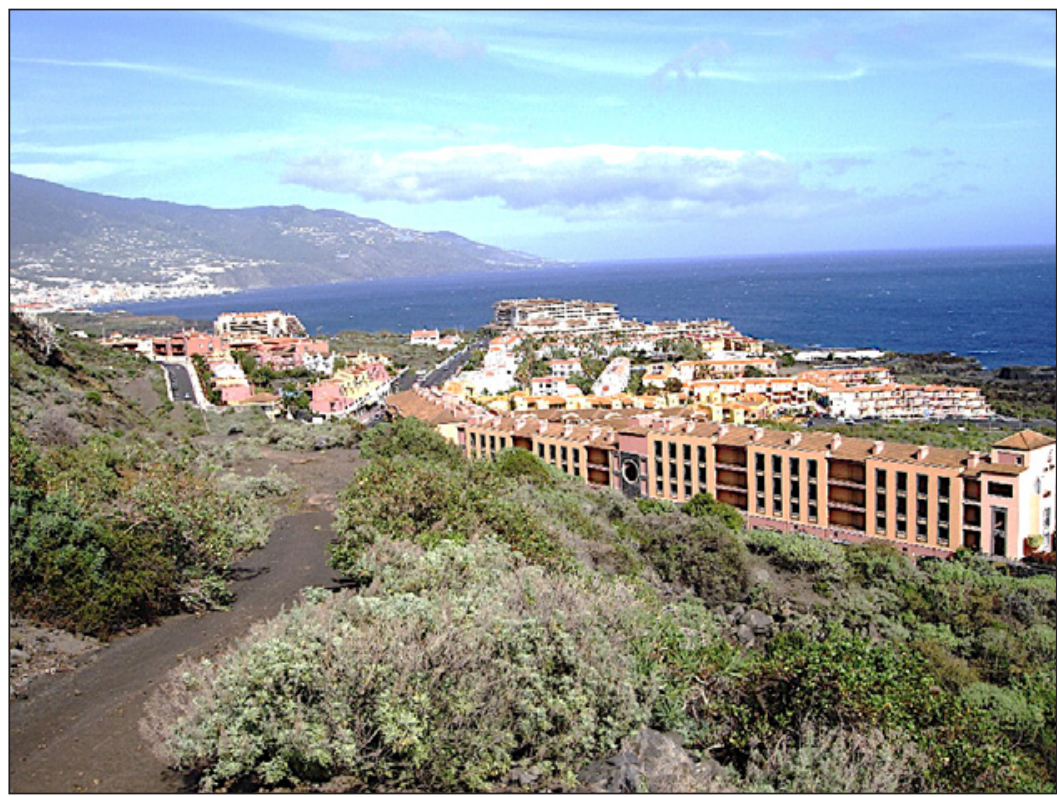

Figura 5

HOTEL EN CERCA VIEJA (FUENCALIENTE)

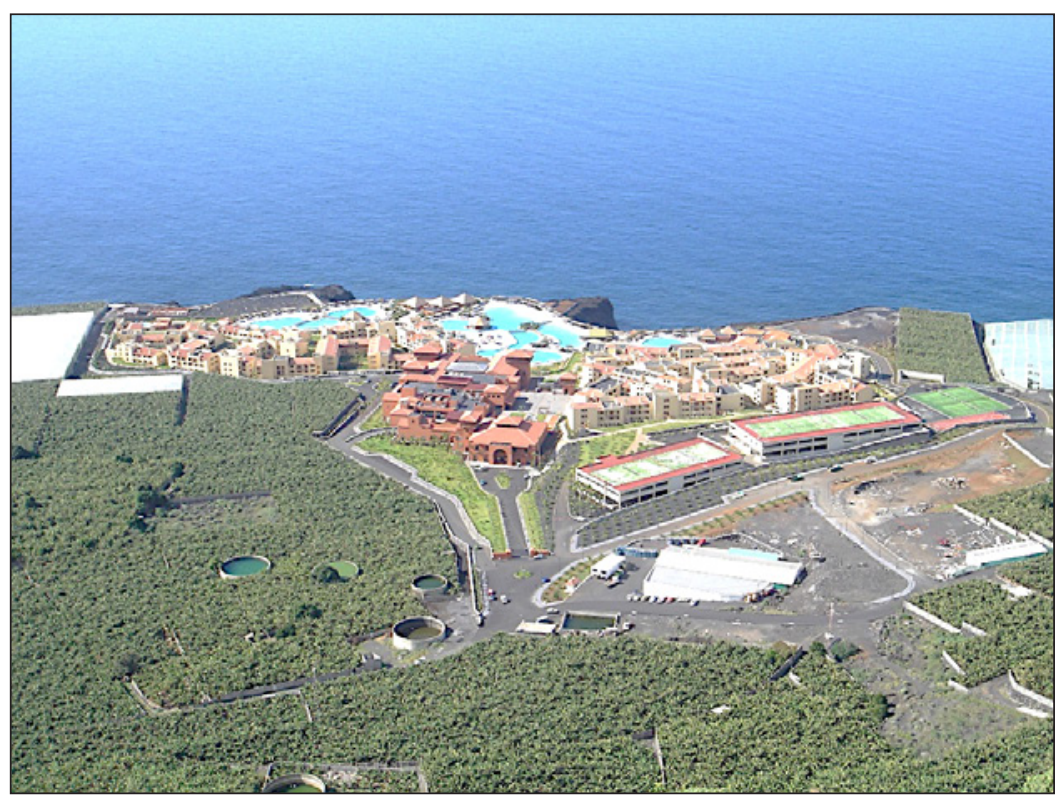




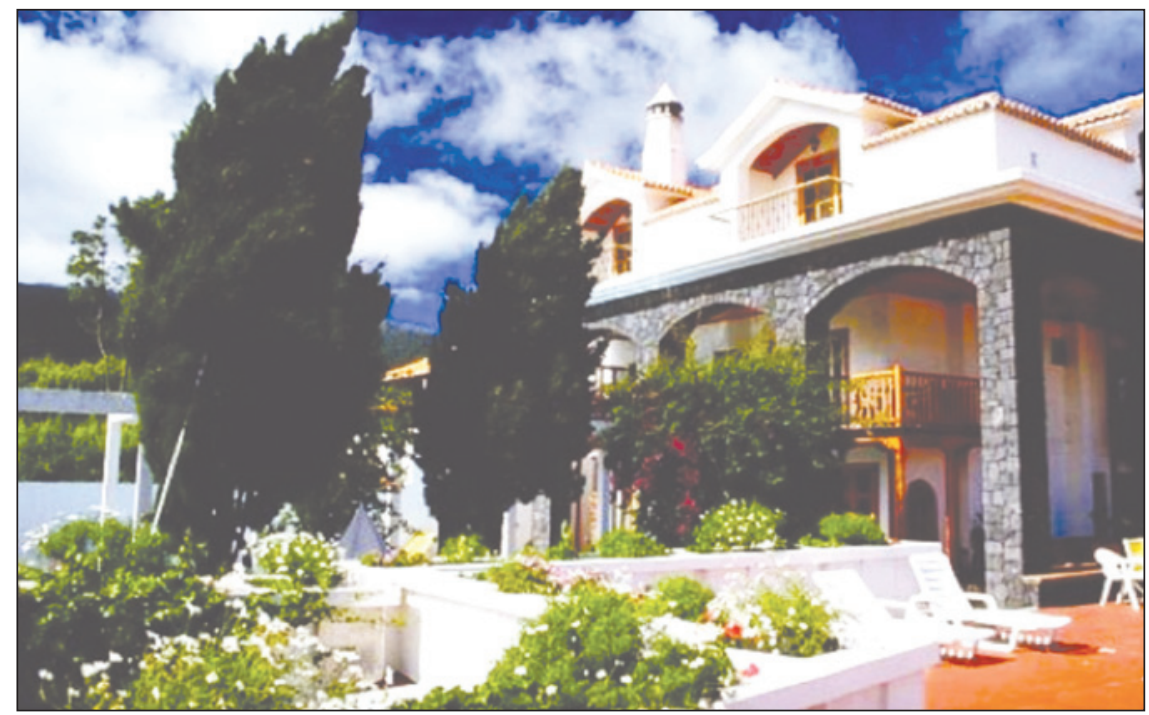

\section{LOS FUTUROS USOS TURÍSTICOS DE LA RESERVA DE LA BIOSFERA DE LA PALMA}

La apuesta por el turismo como estrategia de desarrollo sostenible se ha concretado a través del Decreto 95/2007, de 8 de mayo, por el que se aprueba definitivamente, de modo parcial, el Plan Territorial Especial de Ordenación de la Actividad Turística de la isla de La Palma. Posteriormente, el Plan Insular de Ordenación de La Palma, en vigor desde el 2 de abril de 2011, recoge y asume los usos turísticos sectorizados allí expuestos.

En el Plan Territorial se propone un modelo de desarrollo turístico diferente al que ha caracterizado a otras islas del Archipiélago, más en línea con el turismo de masas. La capacidad máxima de la carga alojativa y el carácter sostenible de las actuaciones turísticas, con una consideración especial del medio ambiente en la toma de decisiones, son los dos pilares en que se basa la apuesta por la sostenibilidad del Plan.

De modo más preciso, la estrategia turística de La Palma se articula en dos modalidades conceptuales: formas convencionales de desarrollo turístico, que en el Plan se consideran necesarias para la conformación de un producto-isla, y turismo de espacios naturales y rurales, que se basan en la gestión de los recursos naturales y autóctonos. En su conjunto, la estrategia ha de garantizar la calidad e integridad de la experiencia turística, el respeto a la capacidad de carga, la valoración activa de la cultura, el desarrollo de una oferta compuesta por pequeños operadores, la gestión local, la formación de todos los agentes y la integración del desarrollo y de la conservación, entre otras características citadas.

En coherencia con estos planteamientos, en el Plan se plantea un incremento moderado en el número de nuevas plazas de alojamiento, representando hasta 2020 unas 11.366 plazas que se suman a las 14.134 ya existentes en 2005. Esto genera para el año horizonte una capa- 
cidad de carga moderada de unos 18.251 turistas por día como promedio, lo que supone el $20 \%$ de la población estante (aquella que reside en la isla) y una densidad media de 160 personas por $\mathrm{km}^{2}$. Estas cifras parecen indicar que estamos ante una previsión de nuevas camas relativamente baja, al menos en términos comparativos con la oferta de las islas orientales y centrales de Canarias.

La propuesta estableció además una prudente temporalización (tabla 3), cronograma además que no se ha cumplido ya que en la actualidad no se ha alcanzado el número de camas previsto en la Isla debido sobre todo a la crisis económica y a la demora hasta 2011 en la aprobación del Plan Insular de Ordenación de La Palma.

Tabla 3

PROGRAMACIÓN DE CAMAS TURÍSTICAS EN LA PALMA (2005 - 2020)

\begin{tabular}{|l|c|c|c|}
\hline Años & Plazas limite & Años & Plazas límite \\
\hline 2005 & 14.134 & 2013 & 23.460 \\
\hline 2006 & 15.903 & 2014 & 23.926 \\
\hline 2007 & 17.550 & 2015 & 24.313 \\
\hline 2008 & 19.017 & 2016 & 24.643 \\
\hline 2009 & 20.276 & 2017 & 24.926 \\
\hline 2010 & 21.329 & 2018 & 25.163 \\
\hline 2011 & 22.192 & 2019 & 25.356 \\
\hline 2012 & 22.892 & 2020 & 25.500 \\
\hline
\end{tabular}

Fuente: Decreto 95/2007, de 8 de mayo, por el que se aprueba definitivamente, de modo parcial, el Plan Territorial Especial de Ordenación de la Actividad Turística de la isla de La Palma.

Las actuaciones turísticas propuestas hasta el año 2020 se distribuyen por casi todo el territorio insular, con lo que se evita la máxima concentración de la oferta en determinados espacios turísticos, que es uno de los principales problemas de las otras islas. Se establecieron cinco zonas diferenciadas con el fin de repartir la oferta turística. El 59,8\% de la isla, que corresponde con los espacios de mayor valor ecológico y paisajístico, se calificó como espacio exento. Las otras cinco, denominadas como de turismo imbricado (39,9\%), corresponden con los ámbitos costeros y de medianías de casi toda la isla. El 0,22\% se reservó para usos turísticos convencionales.

Las zonas 1 y 2, en el centro-oeste de la isla (municipios de Los Llanos de Aridane, Tazacorte y El Paso) y en centro-este (Santa Cruz de la Palma, Breña Baja, Breña Alta, Mazo y Fuencaliente) serán las que albergarán el mayor número de plazas, unas 12.000 y 8.087 respectivamente, entre las existentes y las proyectadas. El resto (noreste, nortenoreste y sur) contendrá entre 1.500 y 2.400 plazas respectivamente. Se supone que con este modelo, los beneficios que genera la actividad turística, tenderán también a dispersarse en el territorio, con lo que se evitarán desequilibrios territoriales importantes, ya que uno de los fenómenos espaciales más destacados es el enorme despegue demográfico de los territorios meridionales de la Isla, frente al envejecimiento y retroceso de la renta de los territorios septentrionales. Al mismo tiempo, excluyendo la zona 3 (Fuencaliente) que se 
considera con una capacidad de carga agotada, el resto albergará un número de plazas que supone sobre el 70\%, en el caso de las dos zonas centrales, y el 45\%, en el de las dos zonas del norte, de la capacidad máxima de acogida.

Las actuaciones previstas en estas cinco zonas se clasifican de tres tipos: el primero lo constituye 13 actuaciones convencionales, es decir, constitutivas de núcleo urbano de uso turístico, previstas fundamentalmente sobre suelo urbanizable sectorizado ordenado y no sectorizado, y en menor medida sobre suelo urbano consolidado y no consolidado. Es decir, que salvo las 900 nuevas plazas previstas en Finca Amado en Breña Baja y El Puerto de Tazacorte, el resto son expansiones o creaciones de nuevos núcleos turísticos. En total, con esta modalidad se crearán 6.282 nuevas plazas (tabla 4).

Tabla 4

ACTUACIONES TURÍSTICAS PREVISTAS EN LA PALMA HASTA 2020 A AÑADIR A LAS QUE ESTÁN EN SERVICIO

\begin{tabular}{|c|c|c|c|}
\hline \multicolumn{4}{|c|}{ Actuaciones Específicas Previstas (AEP) } \\
\hline Enclave & Municipio & Máx camas & Observaciones \\
\hline La Hiedra & El Paso & s.d. & Mínimo hoteles de 4 estrellas (SR)* \\
\hline Tacande & El Paso & s.d. & Mínimo hoteles de 4 estrellas (SR)* \\
\hline Los Quemados & Fuencaliente & 80 & Mínimo hoteles de 5 estrellas (SR)* \\
\hline La Alegría & Breña Alta & s.d. & Mínimo hoteles de 5 estrellas (SR)* \\
\hline Don Pedro & Garafía & 80 & Mínimo hoteles de 4 estrellas (SR)* \\
\hline Las Lomadas & S. A. y Sauces & s.d. & Mínimo hoteles de 4 estrellas (SR)* \\
\hline San Andrés & S. A. y Sauces & s.d. & Mínimo hoteles de 4 estrellas (SR)* \\
\hline \multicolumn{4}{|c|}{ Actuación Convencional Propuesta (ACP) } \\
\hline La Cangrejera & Villa de Mazo & 500 & Mínimo hoteles de 4 estrellas (SUNST)* \\
\hline Balcones Mazo & Villa de Mazo & 500 & Mínimo hoteles de 5 estrellas (SUNST)* \\
\hline Finca Amado & Breña Baja & 400 & $\begin{array}{l}\text { Mínimo hoteles de } 4 \text { estrellas y } \\
\text { apartamentos de } 3 \text { estrellas (SUCYUSO)* }\end{array}$ \\
\hline Los Dragos & Breña Baja & 417 & Mínimo hoteles de 4 estrellas (SUSOT)* \\
\hline Martín Luis & Puntallana & 480 & Mínimo hoteles de 4 estrellas (SUNST)* \\
\hline Santa Lucía & Puntallana & 250 & Mínimo hoteles de 5 estrellas (SUSOT)* \\
\hline La Tahona & Tijarafe & 600 & $\begin{array}{l}\text { Mínimo hoteles de } 5 \text { estrellas y } \\
\text { apartamentos de } 3 \text { llaves (SUNST)* }\end{array}$ \\
\hline Vista Alegre & El Paso & 500 & Mínimo hoteles de 4 estrellas (SUNST)* \\
\hline Las Hoyas & Tazacorte & 900 & Mínimo hoteles de 4 estrellas (SUNST)* \\
\hline Hoyo Verdugo & Tazacorte & 500 & Mínimo hoteles de 4 estrellas (SUSOT)* \\
\hline El Puerto & Tazacorte & 500 & Mínimo hoteles de 5 estrellas (SUNC)* \\
\hline Tazacorte & Tazacorte & 235 & Mínimo hoteles de 4 estrellas (SUNST)* \\
\hline Las Manchas & Los Llanos de A. & 500 & Mínimo hoteles de 4 estrellas (SUNST)* \\
\hline
\end{tabular}




\begin{tabular}{|l|l|l|l|}
\hline \multicolumn{4}{|l|}{ Actuación Estratégica Singular (AES) } \\
\hline Jedey & Los Llanos de A. & 576 & Mínimo hoteles de 4 estrellas (SR)* \\
\hline Las Ledas & Breña Alta & 316 & Mínimo hoteles de 4 estrellas (SR)* \\
\hline Los Canarios & Fuencaliente & 555 & Mínimo hoteles de 4 estrellas (SR)* \\
\hline Barlovento & Barlovento & 269 & Mínimo hoteles de 4 estrellas (SR)* \\
\hline Puntagorda & Puntagorda & 248 & Mínimo hoteles de 4 estrellas (SR)* \\
\hline
\end{tabular}

* SR = Suelo Rústico; SUNST = Suelo Urbanizable No Sectorizado Turístico; SUCYUSO = Suelo Urbano Consolidado Mixto - Urbanizable Sectorizado Ordenado; SUSOT = Suelo Urbanizable Sectorizado Ordenado Turístico; SUNC $=$ Suelo Urbano No Consolidado - Urbanizable No Sectorizado Turístico.

Fuente: Decreto 95/2007, de 8 de mayo, por el que se aprueba definitivamente, de modo parcial, el Plan Territorial Especial de Ordenación de la Actividad Turística de la isla de La Palma.

El segundo tipo lo conforman las denominadas actuaciones estratégicas singulares, esto es, intervenciones aisladas en las que se incluyen instalaciones de ocio junto a unidades alojativas. En total se prevé hasta cinco desarrollos, pero se aconseja que se realicen tan solo cuatro. Estas intervenciones se harán sobre suelos rústicos y computan en total 1.964 plazas, dándose la peculiaridad de que se han distribuido por las cinco zonas de uso turístico imbricado.

Por último, el tercer tipo son las actuaciones específicas previstas. Estas también se situarán sobre suelo rústico, pero en este caso se trata de intervenciones de reducido tamaño dispersas en el territorio y relacionadas con el turismo rural y de naturaleza. La única que se había previsto en el momento de realizar el Plan era la de Don Pedro en Garafía. Las siete actuaciones contempladas y el resto de autorizaciones relacionadas con el turismo rural no suponen más de unas 3.120 nuevas plazas.

En su conjunto, el techo de camas por enclaves de actuación no sobrepasa normalmente las 500, si exceptuamos La Tahona, Jedey y Los Canarios. Solamente supera ostensiblemente esta cantidad el paraje de Las Hoyas, donde se podría admitir hasta 900 camas. El resto, por expresión cuantitativa y densidad previstas, parece adaptarse a un modelo de mayor calidad y baja densidad.

Por último, como marcan las Directrices de Ordenación General y del Turismo en Canarias, los nuevos alojamientos para el turismo tienen que estar sujetos a un incremento de la calidad alojativa, vía aumento de la categoría hotelera y extrahotelera. De este modo, para La Palma se prevé que todas las nuevas construcciones tengan como mínimo una categoría de 4 estrellas, si bien en alguna zona como Finca Amado se pueden desarrollar establecimientos extrahoteleros de la máxima calidad dentro de su categoría, esto es, de 3 estrellas. Habría que destacar además, que en 6 de las actuaciones previstas sólo se permiten hoteles de la máxima categoría (5 estrellas), lo que representan en su conjunto unas 2 mil camas aproximadamente (en torno a un $20 \%$ de las nuevas plazas previstas)

\section{V. ¿UN MODELO TURÍSTICO PROPIO DE UNA ISLA RESERVA DE LA BIOSFERA?}

No podemos negar la preocupación por la sostenibilidad ecológica y social que desprende la propuesta de desarrollo turístico que hemos expuesto brevemente en el epígrafe anterior. Acorde con ello, se ha llevado a cabo un gran esfuerzo técnico para valorar el 
impacto ambiental y la capacidad de carga de las actuaciones previstas. No obstante, no queremos dejar de apuntar algunas observaciones que nos permita reflexionar si estamos ante un modelo turístico apropiado para una isla que en su totalidad ha sido declarada Reserva de la Biosfera.

1. En primer lugar, y sin entrar en mayores valoraciones sobre la adecuación del número de plazas previsto, en los cálculos de capacidad de carga se parte de algunas premisas que son cuestionables, tales como que el crecimiento turístico no repercutirá en un incremento demográfico significativo de la isla. El modelo económico del Plan Territorial apuesta por la gestión local y la implicación de la población palmera en el desarrollo turístico y, sin embargo, la mayor parte de las plazas alojativas corresponde con unidades de explotación de una dimensión tal que requerirán probablemente de la llegada de capitales y de mano de obra desde el exterior para llevarse a cabo.

2. En segundo lugar, a pesar de la apuesta por un turismo basado en los recursos territoriales y culturales de la isla, se propone un modelo híbrido de ocupación (nuevos núcleos turísticos y plazas alojativas dispersas) y, sobre todo, se introducen equipamientos de ocio que no parecen estar relacionados con la tradición cultural palmera. Nos referimos a los enclaves que se pretenden desarrollar en suelo rústico en torno a campos de golf y a nuevos puertos deportivos.

En relación con los nuevos enclaves vinculados al golf, estas actuaciones suponen un consumo de espacio importante en áreas naturales o agrícolas de cierto valor, en algunas ocasiones colindantes con las zonas de exclusión y en otras insertas en áreas protegidas, como el caso de la actuación estratégica singular de Jedey en Los Llanos de Aridane, que se pretende llevar a cabo en el Paisaje Protegido de Tamanca. En todos los casos, el impacto paisajístico va a ser importante, al tratarse de operaciones aisladas en un entorno poco urbanizado. Además, la construcción de los campos de golf va a implicar la edificación de hasta tres nuevos hoteles por enclave y, por tanto, la consolidación de núcleos urbanos que, en el futuro, pueden facilitar el desarrollo de nueva oferta. Como mínimo se prevé para cada una de las actuaciones la reserva de 60 Has para el campo y una instalación hotelera de 200 plazas pero se permite una mayor carga alojativa para hacer viable la explotación turística del campo, de tal modo que las actuaciones pueden suponer desde las poco más de 60 Has de Barlovento hasta las 150,8 de Jedey. Y, por último, las intervenciones van a suponer la revalorización del suelo rústico del entorno, repercutiendo probablemente en la pervivencia de la actividad agrícola.

En los mismos términos nos podemos expresar en relación con los equipamientos marítimos previstos. La isla de La Palma cuenta con 340 atraques deportivos distribuidos entre Tazacorte (165) y la Marina La Palma en San Cruz de la Palma (180). El Plan Director del Paseo Marítimo de Santa Cruz de La Palma ya incluyó la construcción de dos puertos deportivos al norte y sur de la ciudad, dentro de una propuesta ambiciosa que incluye una playa artificial, museo de mar, escuela de vela, paseo marítimo y plazas alojativas. A estas plazas, el Plan Territorial Especial de Ordenación Turística añade otras en el nuevo puerto deportivo de Puerto Naos (150 atraques) y el nuevo Parque Marítimo de los Cancajos (110, que finalmente serán 145), además de la reconversión de Puerto Espíndola en muelle deportivo. Estas infraestructuras impulsarán el crecimiento de los dos núcleos turísticos consolidados de Los Cancajos y Puerto Naos y propiciarán una mayor ocupación del litoral. Como en el caso del golf, el debate no se centra en el número de amarres, sino en el modelo turístico que 
suponen y en el impacto ambiental que genera. En este sentido, la peculiaridad que introduce la condición de Reserva de la Biosfera respecto a otros destinos insulares es una mayor contención en el crecimiento y la adopción de un modelo de ocupación más disperso. Respecto al modelo turístico, no existen diferencias significativas entre el propuesto para La Palma y los de las restantes islas del Archipiélago.

3. En tercer lugar, es necesario reflexionar si el modelo de ocupación turística que se propone, caracterizado por la dispersión territorial de sus actuaciones, no supondrá multiplicar las afecciones paisajísticas. Se ha optado por la dispersión como una estrategia de distribución territorial de los beneficios y de minimización de los impactos ambientales y paisajísticos. Sin embargo, hay que tener presente que el tamaño de muchas intervenciones, potenciará la mejora o creación de infraestructuras y estimulará probablemente el crecimiento residencial.

4. Por último, habría que preguntarse qué es lo que decidirán los políticos y gestores de la planificación cuando se llegue a la mítica cifra de las 25.500 camas planificadas para el año 2020. Sería absurdo pensar que ésta va a ser una meta inamovible. ¿La capacidad de acogida de la isla permitirá entonces nuevos crecimientos?

\section{CONCLUSIONES}

En definitiva, la Reserva de la Biosfera le proporciona a la Isla un sello de calidad ambiental, susceptible de ser usado desde una óptica sostenible por la actividad económica, tanto el turismo como la agricultura y la ganadería. Desde este punto de vista, los valores que presenta la Isla, como por ejemplo sus paisajes físicos y humanos, el importante número de endemismos, el notable patrimonio histórico, en especial de sus espacios urbanos, convierten a La Palma en un territorio merecedor de este galardón de la UNESCO, garante además del compromiso con la sostenibilidad de su territorio.

No obstante, el escaso dinamismo demográfico, sobre todo relacionado con la emigración y el acelerado envejecimiento, demuestra una debilidad estructural importante vinculada a una economía agraria que no proporciona una renta turística más alta como en las islas centrales y más orientales del Archipiélago canario. La alternativa pues, entre otras, debería ser la apertura de la Isla hacia un turismo no masivo como en el resto de las Islas, aprovechando sus notables recursos y su condición de Reserva de la Biosfera.

En relación con lo anterior tenemos que gran parte de los usos turísticos de la Isla se intentan vincular a las casas y viviendas rurales, ligado a la creciente actividad del senderismo, cuya expresión máxima es la Transvulcania, una carrera de larga distancia que se celebra anualmente en la Isla y que está considerada como la ultramaratón de montaña más dura de las Islas Canarias, y una de las más importantes de España, con un desnivel de más de 8.500 metros.

No obstante, el Plan Territorial Especial de Ordenación de la Actividad Turística de la isla de La Palma (2007) y el Plan Insular de Ordenación de La Palma (2011), se decantan por una dispersión de los usos turísticos por toda la Isla y aunque estos suelos ya están conformados en su mayor parte como urbanos con fines turísticos, algunos de ellos como los de Finca Amado, Santa Lucía, La Tahona, Hoyo Verdugo, etc., se localizan como auténticas islas dentro de espacios rurales. Bien es verdad que estas actuaciones turísticas no sobre- 
pasan en su mayor parte las 500 camas cada una de ellas, pero la baja cantidad de camas no excluye el impacto visual que causan, ya que algunas de éstas se ubican en auténticos espacios emblemáticos.

Por otro lado, la apuesta del Plan Territorial Especial de Ordenación Turística supone que la isla alcanzará las 25.500 camas en el año 2020. Indudablemente, la intensificación del uso turístico permitirá que se mantengan cifras inferiores a las que se presentan en Lanzarote, Fuerteventura, Gran Canaria o Tenerife, pero, deberíamos considerar también la capacidad de acogida del medio natural, social y cultural de una isla que es Reserva de la Biosfera para valorar la importancia real de las magnitudes. En otras palabras, deberíamos plantearnos si el incremento que se propone es asumible por el sistema insular sin que se produzca un cambio drástico.

Por último, algunos aspectos del nuevo modelo, como la construcción de hasta cinco campos de golf en suelo rústico no encaja del todo, a nuestro juicio, con lo que debe ser la conservación y la puesta en marcha de un modelo de desarrollo socioeconómico sostenible en La Palma. No parece que el desarrollo de esta actividad sea coherente con los principios propios que inspiran la declaración de Reserva de la Biosfera y por otro lado, el impacto paisajístico, el consumo de suelo, agua y el aumento en el número de camas que va a implicar será elevado.

En definitiva, el modelo territorial que se propone en la isla puede que detenga el proceso de pérdida de efectivos poblacionales, que favorezca la llegada de población e incida en cierto rejuvenecimiento. Asimismo, puede que suponga un crecimiento de la actividad económica y de la renta de la población. Sin embargo, creemos que es un modelo que ni se ajusta a las características propias de la isla, ni al concepto de desarrollo sostenible propio de las Reservas de la Biosfera.

\section{BIBLIOGRAFÍA}

ADER - LA PALMA (Asociación Insular de Desarrollo Rural de La Palma (2007). Informe interno sin publicar.

BILBAO, A. (2007): «Los núcleos rurales en el País Vasco como precursores de la dispersión urbana: la reserva de la biosfera de Urdaibai» Ciudad y Territorio. Estudios Territoriales, $\mathrm{n}^{\mathrm{O}}$ 151. Madrid, 83-101.

BUSTOS, M.L. (2006): «Crisis, recuperación y cambios en las áreas rurales regresivas» Revista de la facultad de Ciencias Económicas. Año IV n 14 Universidad de Zaragoza. Zaragoza, 149-158.

DEL CANTO FRESNO, C. (2000): «Nuevos conceptos y nuevos indicadores de competitividad territorial para las áreas rurales». Anales de Geografía de la Universidad, $\mathrm{n}^{\circ} 20$, 69-84.

DUARTE, R. (2000): «Los programas europeos Leader: Una apuesta por el desarrollo rural» Revista de la facultad de Ciencias Económicas. Año IV n 14 Universidad de Zaragoza. Zaragoza, 149-158.

GARCÍA RODRÍGUEZ, J. L. (1983): La población del Valle de Aridane en La Palma (1857 - 1980). Ed. Secretariado de Publicaciones. Colección Viera y Clavijo, nº V. Santa Cruz de Tenerife, $236 \mathrm{p}$. 
GOBIERNO DE CANARIAS (2003): Planes de uso y gestión de los espacios naturales protegidos. Santa Cruz de Tenerife.

GOBIERNO DE CANARIAS (2005): Plan Director de Actuaciones para el desarrollo del medio rural en las medianías de Canarias. Santa Cruz de Tenerife.

GOBIERNO DE CANARIAS (2007): Programa de desarrollo rural de Canarias 20072013. Santa Cruz de Tenerife.

GOBIERNO DE CANARIAS (2008): Plan Territorial de empleo 2008-2013. Santa Cruz de Tenerife.

GOBIERNO VASCO (2008): Programa de desarrollo rural del País Vasco 2007-2013. Vitoria.

GÓMEZ OREA, D. (2002): Ordenación Territorial. Ediciones Mundi Prensa. Madrid.

HERNÁNDEZ LUIS, J. Á. (1993): Transporte y red viaria en la isla de La Palma, Ed. Universidad de Las Palmas de Gran Canaria y seis instituciones, Las Palmas de Gran Canaria.

ISTAC (2010): Padrón de Habitantes de 2010. Gobierno de Canarias. Las Palmas de Gran Canaria.

MÉNDEZ, R. (1994): «Sistemas productivos locales y políticas de desarrollo rural» Revista de Estudios Regionales, $\mathrm{n}^{\circ}$ 39, 93-114.

MOSCA, J. y RAMOS, E. (1994): «Algunas reflexiones sobre el desarrollo rural y los patrones de acumulación». Estudios Regionales, n 38. Madrid, págs. 81-108.

PARREÑO CASTELLANO, J.M. Y DÍAZ HERNÁNDEZ, R. (2010): «La ordenación territorial, urbanística y de los espacios naturales protegidos y el modelo territorial en la Comunidad Autónoma de Canarias (1982-2009)». Cuadernos Geográficos de la Universidad de Granada, 47, 429-451.

REGIDOR, J. G. (2000): El futuro del medio rural en España. Consejo Económico y Social. Madrid.

\section{RECURSOS WEBS}

http://www.piolp.es/

http://www.lapalmabiosfera.es/

http://www.aderlapalma.org/

http://www.lapalmaturismo.com/

http://www.senderosdelapalma.com/ 
